Original Research Paper

\title{
Short-Term Effects of Aroma Therapeutic Herbal Steam on Heart Rate Variability and Stress
}

\author{
Peerada Damapong and Pongmada Damapong \\ College of Allied Health Science, Suan Sunandha Rajabhat University, Bangkok 10300, Thailand
}

\author{
Article history \\ Received: 13-07-2018 \\ Revised: 19-08-2018 \\ Accepted: 3-09-2018 \\ Corresponding Author: \\ Pongmada Damapong \\ College of Allied Health \\ Science, Suan Sunandha \\ Rajabhat University, \\ Bangkok 10300, Thailand \\ Email: Pongmada.da@ssru.ac.th
}

\begin{abstract}
This experimental research aims to examine the short-term effects of aroma therapeutic herbal steam on heart rate variability and stress. In this experimental study, the participants were 60 diabetic patients with stress who had rated their stress in the Suang-prung Stress Test-20 at the level of 3 or higher. They were divided into the treatment group $(\mathrm{n}=$ $30)$ and the control group $(\mathrm{n}=30)$ using a simple random sampling method. The former was administered aroma therapeutic hot steam, whereas the latter received standard conventional medical care. Both before and after the experimental period, both groups were assessed in terms of their Heart Rate Variability (HRV). The within-group HRV values, namely SDNN, RMS-SD, LF and HF, for both the treatment and the control groups preand post-treatment differed significantly $(p<0.05)$. Additionally, when the between-group values post-treatment were considered, the SDNN, RMS$\mathrm{SD}$ and LF values for the treatment group were significantly higher than those for the control group $(\mathrm{p}<0.05)$. Based on the findings, it can be concluded that aroma therapeutic hot steam will interact with factors triggering stress and enhance parasympathetic nervous system function, thereby reducing stress levels. This is proof that the treatment should improve the conditions of diabetic patients suffering from stress.
\end{abstract}

Keywords: Aroma Therapeutic, Herbal Steam, Heart Rate Variability (HRV), Stress

\section{Introduction}

Stress is a common psychological problem resulting from the reaction of the body, mind and thought to internal and external stimuli, including individuals, feelings, circumstances, or environments. The failure to adjust to such internal and external stimuli can contribute to stress and worsen one's ability to strike a balance in life.

According to a survey on incidents or illnesses causing stress and mental problems in 21 countries around the world conducted by the World Health Organization (WHO), stress is attributable to witnessing violence $(21.8 \%)$, experiencing interpersonal violence (18.8\%), accidents (17.7\%), exposure to war (16.2\%) and trauma to loved ones (12.5\%) (Stein et al., 2010). In addition, physical abnormally, such as disability, chronic illness, or incurable personal illness can all lead to stress. If left untreated for a long period, such conditions may have adverse consequences on both the body and the mind.

Among the above stress factors, diabetes is one of the most prevalent and critical personal illnesses both in Thailand and globally. WHO statistics (WHO, 2016) reveal that the number of adults suffering from diabetes around the world rose from 108 million in 1980 to 422 million in 2014, with more than 1.5 million deaths in 2012 alone. This was accompanied by a jump in the agestandardized global prevalence rates from $4.7 \%$ to $8.5 \%$ in the last decades, especially in low- and middle-income countries. The figures reflect a growth in the incidence of such associated risk factors as obesity. In Thailand, the public health statistics during 2010-2014 similarly indicate a continually increasing number of diabetic patients (Strategy and Planning Division, 2015) with the prevalence rates in males falling from $6.4 \%$ to $6.0 \%$ and in females going up from $7.3 \%$ to $7.7 \%$ (Bureau of non communicable diseases, 2015) Diabetic patients experience stress from the illness in numerous ways. In order to prevent complications and ensure good life quality, they have to be very disciplined in receiving treatment, watching their diet, taking medication, doing exercise and controlling their blood sugar, blood pressure and weight. As a result, they may experience abrupt changes in their lifestyle, for instance, from eating an unlimited amount of the food they like to strict diet 
control. In addition, patients with this illness tend to suffer from a strong feeling of insecurity because of the uncertainty associated with its complications and increasing severity. All this can in turn lead to mental issues and even worsen the life quality of diabetic patients.

In response to stress-related problems, WHO has devised the Guidelines for the Management of Conditions Specifically Related to Stress (WHO, 2013), encompassing both modern medicine and alternative medicine, including naturopathy, herbal steam and aromatherapy (Hozumi et al., 2017). The guidelines provide a basis for the present study. The aroma therapeutic herbal steam has relaxing effects as a result of the heat, herbs and aroma therapeutic used in the process, apart from its benefits to the respiratory system. Thus, it is of interest to examine the effectiveness of aroma therapeutic herbal steam for reducing stress in diabetic patients.

\section{Objective}

\section{General Objective}

To examine the short-term effects of aromatherapeutic herbal steam on diabetic patients with stress in Chaiyaphum, a province in the northeast of Thailand.

\section{Specific Objective}

- To examine the Heart Rate Variability (HRV) of diabetic patients with stress receiving aromatherapeutic herbal steam treatment

- To compare the short-term effects of aromatherapeutic herbal steam and standard care on diabetic patients with stress

\section{Materials and Methods}

This experimental study was conducted in Phakdi chumphon district, Chaiyaphum province in the northeast of Thailand. The inclusion criteria were diabetic patients aged 35-85 who had rated their stress in the Suang-prung Stress Test-20 (SPST-20) at the level of 3 or higher. The excluded patients were those who were having a fever higher than $38^{\circ} \mathrm{C}$; were suffering from all kinds of malignant infectious disease; were having such personal illness as kidney disease, heart disease, epilepsy, severe asthma, severe respiratory tract infection; were female during menstruation accompanied by a fever; were exhibiting symptoms of inflammation caused by wounds; were experiencing a severe headache accompanied by nausea; and had a history of allergy to herbs. The 60 patients having fulfilled the inclusion criteria were then divided into the treatment group ( $\mathrm{n}=$ $30)$ and the control group $(n=30)$ using a simple random sampling method. The treatment group received aroma therapeutic herbal steam, while the control group was administered standard conventional medical care. Prior to and after the experimental period, both groups were assessed in terms of HRV.

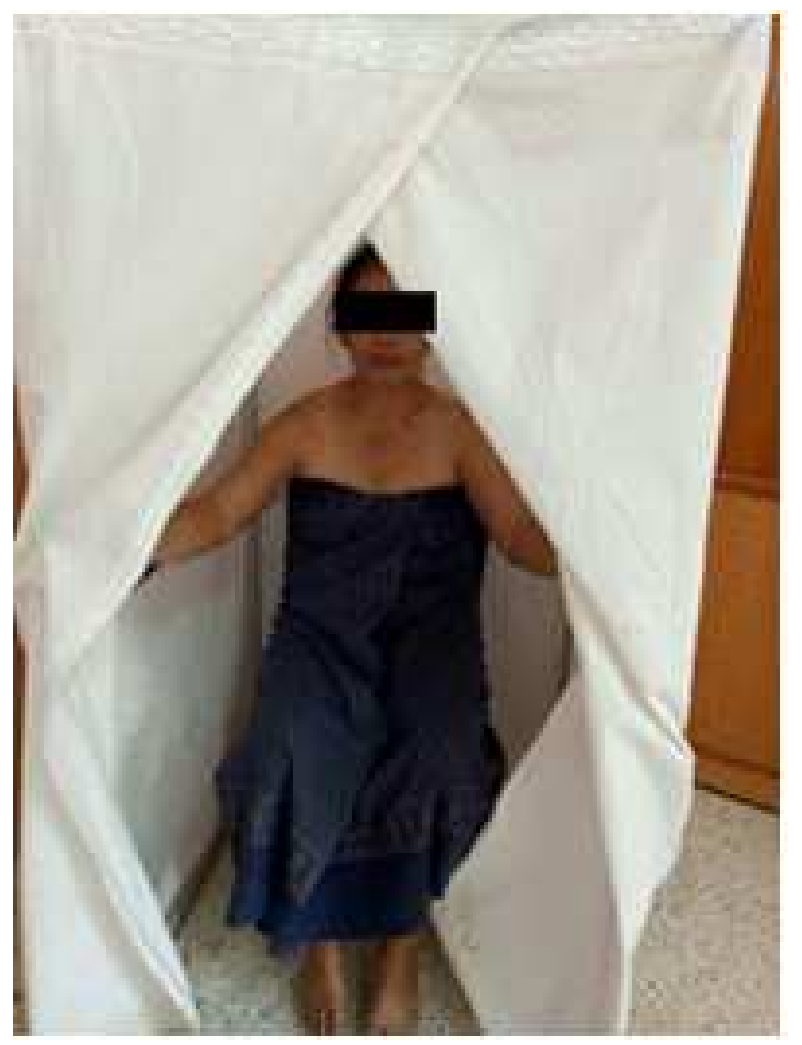

Fig. 1: Aroma therapeutic herbal steam administered to the treatment group

The treatment group was administered therapeutic herbal steam every other two days for a week. Each session lasted $30 \mathrm{~min}$ and was divided into two 15minute sessions with a 5 min break in-between, as shown in Fig. 1. In contrast, the control group received standard conventional medical care. The herbal formula used in this research has relaxing effects and helps to alleviate body aches. It is comprised of an essential oil drug class, including Bengal roots, arrowroots, curcumin and kaffir lime peels and a stress relief drug class, including jasmine, Bullet wood flower, Ironwood flower and Negkaser flower.

The research project was explained to the volunteers. Those diagnosed with stress by physicians were then asked to complete questionnaires surveying personal data and stress levels. The 60 volunteers having fulfilled the inclusion criteria were asked to show their intent to participate in the study by signing a consent form. After that, the participants were divided into the treatment group $(\mathrm{n}=30)$ and the control group $(\mathrm{n}=30)$ using a simple random sampling method. The former was administered aroma therapeutic herbal steam, whereas the latter received standard care. As for the treatment group, the aroma therapeutic herbal steam was conducted by Thai traditional medical doctors or applied Thai traditional medical doctors possessing a 
medical license with more than three years of experience. The administration of the herbal steam took place every other two days for a week. Each session lasted $30 \mathrm{~min}$ and was divided into two 15 min sessions with a 15 min break in-between. In contrast, the control group was provided standard care for diabetic patients with stress by conventional medical doctors. The participants in the two groups were assessed in terms of stress levels and HRV both pre- and post-treatment. The research instruments were the SPST-20 for measuring stress levels and the Heart Rhythm Scanner PE (Biocom Technologies, USA) for measuring HRV. The demographic characteristics of the patients were analyzed using descriptive statistics. The within-group variables were analyzed using the paired t-test and the between-group variables were analyzed using the ttest at the significance level of 0.05 .

\section{Results and Discussion}

Comparisons of the pre- and post-treatment withingroup HRV values indicated significant increases in SDNN, RMS-SD, LF and HF for both the treatment and the control groups $(\mathrm{p}<0.05)$. Additionally, comparisons of the post-treatment between-group HRV values revealed that the SDNN, RMS-SD and LF values for the treatment group were significantly higher than those for the control group $(\mathrm{p}<0.05)$, as shown in the Table 1 and 2.

The present findings suggest the positive effects of alternative medicine on parasympathetic nervous system function and stress relief, in line with the results reported in previous research. Matsumoto et al. (2017; Yang et al., 2015; Damapong et al., 2016; Toro-Velasco et al., 2009; Matsumoto et al., 2017) investigated the lavender-like effects of yuzu, a citrus fruit, on premenstrual emotional systems. They found that $10 \mathrm{~min}$ inhalation of yuzu contributed to a significant drop in heart rates and a significant rise in HRV high frequency power $(\mathrm{p}<0.05)$. In addition, their analysis of the Profile of Mood States (POMS) test results showed that the therapeutic effects of yuzu could continue to significantly reduce three negative subscales, namely tension-anxiety, angerhostility and fatigue-common premenstrual symptoms for as long as 35 min post-treatment $(\mathrm{p}<0.05)$. Yang et al. (2015) compared the efficacy of aroma-acupuncture and aromatherapy in treating agitation caused by dementia, discovering that the treatment group being administered aroma-acupuncture treatment exhibited significantly higher HRV values than the aromatherapy-only group $(p<0.05)$. Based on the results, they explain that the inhalation of aroma therapeutic essential oils can help to relax muscles and in turn alleviate stress.

Table 1: Compare outcome measures within group between before and after the treatment

\begin{tabular}{lllcc}
\hline Outcome & Group & $\begin{array}{l}\text { Baseline } \\
\text { (Mean } \pm \text { SD) }\end{array}$ & $\begin{array}{l}\text { Short-term effectiveness } \\
\text { (Week 1) (Mean } \pm \text { SD) }\end{array}$ & \begin{tabular}{c} 
P-value \\
\hline Heart Rate Variability (HRV)
\end{tabular} \\
- SDNN (Ms) & Treatment & $22.71 \pm 14.89$ & $35.10 \pm 23.45$ & $<0.05^{*}$ \\
& Control & $24.17 \pm 18.55$ & $23.82 \pm 17.56$ & 0.832 \\
- RMS-SD (Ms) & Treatment & $17.92 \pm 15.02$ & $30.06 \pm 25.08$ & $<0.05^{*}$ \\
& Control & $17.77 \pm 15.92$ & $18.95 \pm 13.81$ & 0.231 \\
- LF $\left(\mathrm{ms}^{2}\right)$ & Treatment & $27.16 \pm 21.12$ & $44.42 \pm 34.98$ & $<0.05^{*}$ \\
& Control & $25.96 \pm 22.98$ & $27.86 \pm 25.20$ & 0.430 \\
- HF $\left(\mathrm{ms}^{2}\right)$ & Treatment & $22.11 \pm 20.59$ & $36.95 \pm 22.64$ & $<0.05^{*}$ \\
& Control & $18.57 \pm 18.88$ & $27.81 \pm 23.30$ & $0.001^{*}$ \\
- LF/HF $\left(\mathrm{ms}^{2}\right)$ & Treatment & $1.59 \pm 0.68$ & $1.84 \pm 1.28$ & 0.223 \\
& Control & $1.47 \pm 0.68$ & $1.62 \pm 0.89$ & 0.409 \\
\hline
\end{tabular}

Note: $* \mathrm{p}<0.05$ is statistically significant differences as compare between before and after the treatment from baseline

Table 2: Compare outcome measures between before and after the treatment at each assessment time point

\begin{tabular}{|c|c|c|c|c|c|c|}
\hline \multirow[b]{2}{*}{ Outcome } & \multicolumn{2}{|c|}{ Baseline (Mean \pm SD) } & \multicolumn{2}{|c|}{$\begin{array}{l}\text { Short-term effectiveness } \\
(\text { Week 1) }(\text { Mean } \pm \text { SD) }\end{array}$} & \multirow[b]{2}{*}{$\mathrm{t}$} & \multirow[b]{2}{*}{ P-value } \\
\hline & Treatment & Control & Treatment & Control & & \\
\hline \multicolumn{7}{|c|}{ Heart rate variability (HRV) } \\
\hline - SDNN (Ms) & $22.71 \pm 14.89$ & $24.17 \pm 18.55$ & $35.10 \pm 23.45$ & $23.82 \pm 17.56$ & 2.109 & $0.039 *$ \\
\hline - RMS-SD (Ms) & $17.92 \pm 15.02$ & $17.77 \pm 15.92$ & $30.06 \pm 25.08$ & $18.95 \pm 13.81$ & 2.125 & $0.038^{*}$ \\
\hline$-\mathrm{LF}\left(\mathrm{ms}^{2}\right)$ & $27.16 \pm 21.12$ & $25.96 \pm 22.98$ & $44.42 \pm 34.98$ & $27.86 \pm 25.20$ & 2.103 & $0.040 *$ \\
\hline$-\mathrm{HF}\left(\mathrm{ms}^{2}\right)$ & $22.11 \pm 20.59$ & $18.57 \pm 18.88$ & $36.95 \pm 22.64$ & $27.87 \pm 23.30$ & 1.532 & 0.131 \\
\hline$-\mathrm{LF} / \mathrm{HF}\left(\mathrm{ms}^{2}\right)$ & $1.59 \pm 0.68$ & $1.47 \pm 0.68$ & $1.84 \pm 1.28$ & $1.62 \pm 0.89$ & 0.758 & 0.452 \\
\hline
\end{tabular}

Note: ${ }^{*} \mathrm{p}<0.05$ is statistically significant differences as compare between before and after the treatment from baseline 
Damapong et al. examined the short-term effects of Courttype Traditional Thai Massage (CTTM) on pressure pain threshold and pain intensity in patients with Chronic Tension-Type Headache (CTTH) (Damapong et al., 2015), finding that the treatment could significantly alleviate pressure pain and pain intensity $(p<0.05)$. In another study, Damapong et al. conducted a randomized controlled trial on the effectiveness of CTTM vs. amitriptyline in CTTH patients (Toro-Velasco et al., 2009). They similarly discovered that the CTTM group had significantly higher HRV than the amitriptyline group $(\mathrm{p}<0.05)$. Finally, Toro-Velasco et al. investigated the immediate effects of head and neck massage on CTTH patients (Damapong et al., 2015), finding a significant change in HRV interaction time in the treatment group receiving head and neck massage $(\mathrm{p}<0.05)$.

\section{Conclusion}

The present research on the short-term effects of aroma therapeutic herbal steam on the HRV and stress levels of diabetic patients with stress led to several important findings. First, the post-treatment withingroup HRV values, namely SDNN, RMS-SD, LF and $\mathrm{HF}$ increased significantly from the pre-treatment ones for both the treatment and the control groups. In addition, the post-treatment SDNN, RMS-SD and LF values associated with the treatment group were significantly higher than those for the control group. Such findings suggest that the inhalation of essential oils during aroma therapeutic herbal steam can help to relieve stress-related factors, thereby improving parasympathetic nervous system function and alleviating stress, especially in diabetic patients.

\section{Limitations of the Study}

The aroma therapeutic herbal steam involved side effects. That is, some of the volunteers who had never received aroma therapeutic herbal steam before reported experiencing in the dizziness after the first treatment that subsided within one to two hours.

\section{Implications and Recommendations for Further Study}

Based on the present findings, on the short-term effects of aroma therapeutic herbal steam on the HRV and stress levels of diabetic patients could serve as an alternative therapy for the treatment of stress.

Further research should determine long term effects of aroma therapeutic herbal steam because this research was the short-term effects within one week of treatment. Effects of longer period of treatment and longer followup period are not known.

\section{Acknowledgement}

The researchers are very grateful to all participants for contributing important information. Our thanks also go to the Public Health Chief Officer, Public Health Officers, Nurses and the other of Phakdi chumphon district, Chaiyaphum province for the kind guidance, assistance and cooperation.

\section{Funding Information}

This research was supported by National Research Council of Thailand and Suan Sunandha Rajabhat University, Thailand.

\section{Author's Contributions}

Peerada Damapong: Carried out the experimental setup, conception and research design, collected data and evaluation, discussion, conclusion.

Pongmada Damapong: Proof reading, introduction, literature review, system description, experiment and evaluation.

\section{Ethics}

The research was approved by the Human Research Ethics Committee of Sirindhorn College of Public Health Khon Kaen (81/2018, HE610662).

\section{References}

Bureau of non communicable diseases, Ministry of Public Health, Thailand. 2015. Annual report.

Damapong, P., N. Kanchanakhan, W. Eungpinichpong, P. Putthapitak and P. Damapong, 2015. A randomized controlled trial on the effectiveness of court-type traditional Thai massage versus amitriptyline in patients with chronic tension-type headache. Evidence-Based Complementary Alternative Med., 2015: 930175-930175. DOI: $10.1155 / 2015 / 930175$

Damapong, P., N. Kanchanakhan, W. Eungpinichpong, P. Putthapitak and P. Damapong, 2016. Short-term effects of court-type traditional Thai massage on pressure pain threshold and pain intensity in patients with chronic tension-type Headach. Int. J. GEOMATE.

Hozumi, H., S. Hasegawa, T. Tsunenari, N. Sanpei and Y. Arashina et al., 2017. Aromatherapies using Osmanthus fragrans oil and grapefruit oil are effective complementary treatments for anxious patients undergoing colonoscopy: A randomized controlled study. Complementary Therapies Med., 34: 165-169. DOI: 10.1016/j.ctim.2017.08.012 
Matsumoto, T., T. Kimura and T. Hayashi, 2017. Does Japanese citrus fruit yuzu (Citrus junos Sieb. ex Tanaka) fragrance have lavender-like therapeutic effects that alleviate premenstrual emotional symptoms? A single-blind randomized crossover study. J. Alternative Complementary Med., 23: 461-470. DOI: $10.1089 / \mathrm{acm} .2016 .0328$

Stein, D.J., W.T. Chiu, I. Hwang, R.C. Kessler and N. Sampson et al., 2010. Cross-national analysis of the associations between traumatic events and suicidal behavior: Findings from the who world mental health surveys. PLoS ONE, 5: e10574-e10574.

DOI: 10.1371/journal.pone.0010574

Strategy and Planning Division, Ministry of Public Health, Thailand. 2015. Public health statistics.
World Health Organization. 2013. Guidelines for the management of conditions specifically related to stress. Assessment and management of conditions specifically related to stress: MhGAP intervention guide module (Version 1.0).

WHO, 2016. Global report on diabetes. World Health Organization.

Yang, M.H., L.C. Lin, S.C. Wu, J.H. Chiu and P.N. Wang et al., 2015. Comparison of the efficacy of aroma-acupressure and aromatherapy for the treatment of dementia-associated agitation. BMC Complementary Alternative Med., 15: 1-8.

DOI: $10.1186 / \mathrm{s} 12906-015-0612-9$ 